\title{
ESTUDIO LIMNOLÓGICO DEL ECOSISTEMA FLUVIAL AFECTADO POR LOS VACIADOS DEL EMBALSE DE BARASONA
}

\author{
Antoni Palau Ybars \\ Dep. Medi Ambient i Ciències del Sòl (E.T.S.E.A.)Universitat de Lleida. Av. A. Rovira Roure, 177.25198-Lleida.
}

\section{RESUMEN}

Este artículo es un resumen de los principales resultados obtenidos del seguimiento ambiental de los efectos sobre el río a g a abajo. de los ires vaciados totales consecutivos del embalse de Barasona (río Esera, Huesca) en los meses de octubre de 1995.1996 y 1997.

Los electos del vaciado del embalse se concretaron en importantes cambios en la físico-química del agua, especialmente en el oxígeno disuelto. Las poblaciones de macrobentos también se vieron fuertemente afectadas. Las poblaciones de peces del embalse presentaron niveles de mortalidad muy altos. No obstante. todos estos cambios quedaron atenuados aguas abajo y fueron reversibles a corto plazo gracias en parte al lavado del cauce por las creciddas naturales ocurridas después de los vaciados. Otros aspectos clave fueron la gran cantidad de sedimento\movilizados desde el embalse y su baja carga orgánica y concentración de metales pesados.

Palabras clave: Embalses, vaciado total, impacto ambiental

\begin{abstract}
This paper is a summary of the main obtained results in the environmental study of three consecutive flushing of the Barasona reservoir (October of 1995, 1996 and 1997) on the downstream limnological characteristics.

The effects of the full emptying were significant on some physical and chemical variables, specially oxygen and ammonia. Zoobenthos populations were strongly affected, and mortality of fishes flowing from the reservoir was very high during the final phase of water and sediments discharge. These changes were less intense downstream where the return to their former values very was fast because of the washing effect of natural floods. Moreover, the low proportion of organic matter and heavy metals in the reservoir sediment contributed to minimise the flushing effects.
\end{abstract}

Key words: Reservoirs, lotal emptying, environmental impact

\section{INTRODUCCIÓN}

El presente artículo es un resumen del estudio de seguimiento de los efectos del vaciado total del embalse de Barasona, sobre la físico-química del agua y las comunidades de macroinvertebrados bentónicos (macrobentos), en el cauce de los ríos Esera y Cinca aguas abajo del citado embalse (Palau, 1996).

El estudio se inició a finales de 1993 con la caracterización de la situación preoperacional de la zona de estudio antes del primer vaciado (Octubre de 1995); continuó durante el segundo vaciado (Octubre de 1996) y acabó después del tercer vaciado consecutivo (Octubre de 1997). Los datos que se presentan son un extracto de los más interesantes y corresponden fundamentalmente al primer vaciado del embalse (Octubre de 1995)y a los tramos de río más próximos a la presa.

Cabe indicar que en España existen pocos antecedentes sobre estudios de seguimiento y resultados como los que ahora se presentan (Palau y Rivero, 1994). La estrategia de trabajo, así como buena parte de los parámetros y variables clave del estudio se obtuvieron de la experiencia francesa "LIVRE" (Cardinal, 1989) ya que el resto de información encontrada a menudo se presentaba de forma segmentada $\mathbf{y}$ a veces marginal (Grizzle, 1981: Fisher et al., 1982; Garric et al., 1990: Peviani et al., 1996). 


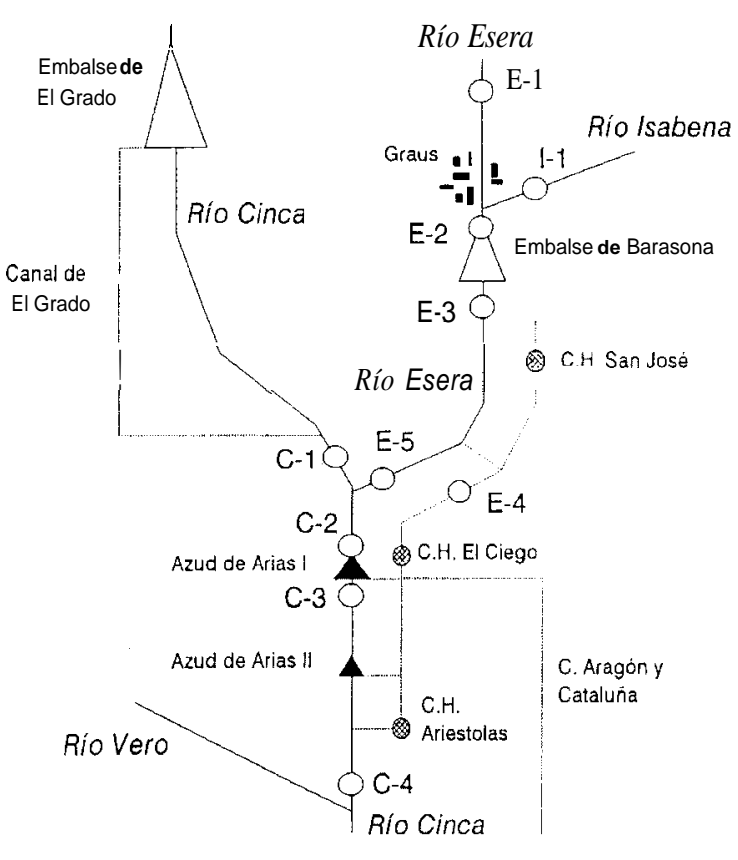

Figura 1. Esquema orientativo de localización del embalse de Barasona, de los sistemas acuáticos asociados y de los puntos de muestreo básicos. Guiding map showing the location of Barasona reservoir, the associated fluvial system and the main sampling places.

\section{MATERIAL Y MÉTODOS}

Para la realización de las diferentes fases del estudio, se utilizó un total de 22 puntos de muestreo situados entre el río Esera a la entrada del embalse, hasta el río Segre en la cola del embalse de Ribarroja, si bien no todos los puntos han sido objeto de una misma intensidad de muestreo. La Figura 1 muestra la red de los 10 puntos básicos de muestreo.

Entre Diciembre de 1993 y Octubre de 1997, se llevaron a cabo un total de 52 campañas de muestreo, distribuidas de forma aperiódica según las fases del estudio. La duración de las campañas fué de un día o de varios y la cadencia de los muestreos desde 4 meses (seguimientos de control) hasta del orden de un minuto (seguimientos durante los vaciados).

En los diferentes puntos establecidos y campañas de muestreo realizadas, se efectuaron análisis físicos y químicos de sedimentos del embalse $(\mathrm{pH}$, $\mathrm{NH}_{4}^{+}, \mathrm{DBO}$, ) y de aguas (temperatura, $\mathrm{pH}$, con- ductividad eléctrica, turbidez, oxígeno disuelto, alcalinidad total, nitrógeno y fósforo inorgánico disuelto, sulfhídrico, DQO, cationes y aniones mayoritarios y algunos metales (hierro, manganeso, cromo VI, cadmio, plomo). Los métodos de análisis y tratamiento de las muestras de agua se obtuvieron de Rodier, (1981) y Stednick, (1991).

En relación al macrobentos se ha estudiado su distribución, su composición taxonómica y características ecológicas (Tachet et al., 1980), las densidades y biomasas, la diversidad y la calidad biológica (índice biótico BMWP'; Alba-Tercedor y Sanchez-Ortega, 1988).

\section{CARACTERÍSTICAS GENERALES DE LOS RÍOS ANTES DEL VACIADO}

El embalse de Barasona se encuentra al NE de la provincia de Huesca, en los términos municipales de La Puebla de Castro (presa) y de Barasona (embalse). Sus dos principales tributarios son, por orden de importancia, el Esera y el Isábena, que representan más del $98 \%$ de la aportación total (unos $845 \mathrm{Hm}^{3} / \mathrm{año}$ ), y sigue un régimen nivopluvial. Las demandas de agua (riegos) se centran entre Marzo y Octubre con máximos en Mayo, Julio y Agosto. El embalse tiene también servidumbres de abastecimiento urbano (Acequia de Estada y Canal de Aragón y Cataluña), así como de aprovechamiento hidroeléctrico (C.H. San José y El Ciego).

Se trata de un embalse poco productivo (mesooligotrófico; Margalef et al., 1976; Morgui et al., 1990), lo que junto con otros factores determina que la cantidad de materia orgánica presente en el sedimento sea muy baja. El grado de mineralización de sus aguas es alto (Armengol et al., 1991). En 1993, Barasona presentaba aproximadamente entre 16 y $18 \mathrm{hm}^{3}$ de sedimentos acumulados, con espesores máximos del orden de 20 a 25 m inmediatamente al lado de la presa.

El tramo del río Esera comprendido entre la presa de Barasona y el río Cinca (un tramo de 6 $\mathrm{Km}$ aproximadamente) presenta un cauce encajado en un estrecho desfiladero rocoso. Hasta el momento del vaciado, no existía un caudal de mantenimiento establecido aguas abajo de la presa, de modo que se alternaban largos periodos de caudales mínimos (alrededor de 80 l/s debido a filtraciones) con crecidas puntuales de regula- 
ción de más de $150 \mathrm{~m}^{3} / \mathrm{s}$. Los caudales mínimos aumentaban de manera sustancial aguas abajo con el retorno de aguas de la Central Hidroeléctrica San José (últimos $4 \mathrm{Km}$. del Esera, aproximadamente).

En circunstancjas normales, las aguas del tramo final del río Esera se caracterizaban por una variabilidad térmica importante $(3-28$ "C) y también en el contenido de oxígeno. Eran aguas de mineralización moderada y con bajas concentraciones de amonio y fósforo inorgánico disuelto. En estas condiciones el macrobentos se encontraba limitado a varios niveles (calidad y cantidad de agua, hábitat, estabilidad, etc.), de ahí que estuviese formado por poblaciones de especies tolerantes y cosmopolitas que alcanzaban una comunidad algo más diversa y estructurada, en los últimos $4 \mathrm{Km}$ del río.

En conjunto el tramo presentaba unas biomasas de macrobentos calificables de bajas (0.03$1.05 \mathrm{~g} \mathrm{C} / \mathrm{m}^{2}$ ) y un índice biótico (BMWP') entre 25 y 95 aproximadamente, con la mayor parte de los valores dentro de las categorías indicadoras de alteración significativa.

Sobre el río Cinca en la zona de estudio, su régimen es nivopluvial atenuado (regulado), con un caudal medio de unos $30 \mathrm{~m}^{3} / \mathrm{s}$ sometido también a fluctuaciones importantes, tanto estacionales (sueltas de regulación de más de $200 \mathrm{~m}^{3} / \mathrm{s}$ ), como horarias (hidropuntas entre 10 y $40-45 \mathrm{~m}^{3} / \mathrm{s}$ ). Todo ello determina un cauce abierto (la antítesis del tramo final del Ésera), con potentes acopios de gravas y cantos rodados muy afectados por explotaciones de áridos, con múltiples canales en forma dendrítica.

Las aguas son, en origen, de buena calidad, menos mineralizadas que las del Esera y con bajas concentraciones de materia orgánica y nutrientes, en especial a la altura de la confluencia del Ésera. Aguas abajo, los vertidos recibidos y sobre todo la entrada del río Vero, junto con el excesivo trenzamiento del cauce, producen una pérdida significativa de la calidad del agua, más apreciable en verano, cuando no se alcanzan temperaturas superiores a $\operatorname{los} 28^{\circ} \mathrm{C}$.

El macrobentos está dominado por taxones tolerantes, indicativos de un cierto grado de alteración del medio, no atribuibles tanto a la calidad del agua como a las modificaciones que sufre el cauce. El índice BMWP' da valores que oscilan entre 23 y 80 , con biomasas entre 0.04 y 5.70 g
$\mathrm{C} / \mathrm{m}^{2}$; valoresdel mismo orden que los registrados en el Ésera, excepto el máximo de biomasa, lo que constituye una buena evidencia de que, en los ríos la calidad y cantidad de agua sin más, aunque indispensables, no siempre son lo único importante.

\section{COMPOSICIÓN FÍSICA Y QUÍMICA DEL AGUA DURANTE EL VACIADO}

El seguimiento de las características físicas y químicas del agua durante los tres vaciados fue, sin duda, la fase de muestreo más intensivo y espectacular del estudio. Duró unas 40 horas, durante las cuales se procedió a la recogida periódica de muestras a diferentes escalas de tiempo a intervalos que iban desde 2 horas hasta unos POcos minutos.

El tramo de río donde más y mejor se ha manifestado la magnitud del vaciado, ha sido Iógicamente el más próximo a la presa ya que aguas abajo se ha dado siempre una creciente atenuación de la dispersión de valores (decantación, aireación, dilución, etc.), en especial a partir del punto en el que el río Ésera desemboca en el río Cinca. Cabe indicar que en el caso del río Cinca, al margen de la carga de sólidos en suspensión, aunque se registraron cambios, no se observaron valores particularmente reseñables para el conjunto de parámetros y variables físico-químicas estudiadas. Los principales cambios en el río Cínca, aún sin ser tampoco reseñables, tenían lugar más bien en los días siguientes a los vaciados. Por esta razón, los resultados que se exponen en este documento se refieren fundamentalmente al punto E-3, el más próximo a la presa. También se incluyen comentarios sobre los resultados obtenidos en otros puntos de los tramos de río estudiados, así como de aspectos que, aún no siendo del ámbito de las características físicas y químicas del agua, también se pudieron percibir. Tal es el caso de las observaciones y evaluaciones orientativas realizadas sobre la población íctica saliente del embalse.

En la Tabla 1 se recoge, a modo de muestra, la relación cronológica del proceso de vaciado correspondiente a 1995 en el tramo final del río Esera.

La Figura 2 indica la evolución orientativa seguida por los caudales salientes durante el pri- 
Tabla I. Cronologia del vaciado. Chronology of the emptying.

\begin{tabular}{|c|c|c|c|c|c|}
\hline \multirow{2}{*}{$\begin{array}{l}\text { Estación } \\
\text { muestreo }\end{array}$} & \multirow{2}{*}{$\begin{array}{c}\text { Distancia } \\
\text { presa }\end{array}$} & \multicolumn{2}{|c|}{ Llegada frente de agua } & \multicolumn{2}{|c|}{ Llegada poso final Iodos } \\
\hline & & Hora real & H. relativa & Hora real & Hora relativa \\
\hline E-3 & $200 \mathrm{~m}$ & $2 \mathrm{~h} 54^{\prime}$ & 6h 04' & 20h So' & 24h 00' \\
\hline E-5 & $6 \mathrm{~km}$ & $3 \mathrm{~h} 40^{\prime}$ & 6h SO' & $22 \mathrm{~h} 20^{\prime}$ & $25 \mathrm{~h} 30$ \\
\hline
\end{tabular}

mer vaciado (1995) y que, en líneas generales (tendencias de variación), es aplicable al resto de años (vaciados de 1996 y 1997), excepto en lo que se refiere a la parte final del vaciado. Así en 1995 se caracterizó por una serie de pulsos alternos de caudal líquido y caudal sólido debido al taponamiento y destaponamiento continuado de los conductos de los desagües de fondo, mientras que en 1996 y 1997 fue mucho menos fluctuante.

Un aspecto a tener en cuenta en la interpretación de los resultados es el estado preoperacional del punto E-3 (aguas abajo de la presa), en particular antes del primer vaciado (1995), cuando el cauce estaba formado, como ya se ha comentado, por un rosario de pozas sujetas a unos caudales muy bajos.

En la Figura 3 se representan los datos del punto E-3 referidos al oxígeno disuelto como una de las variables más relevantes durante el seguimiento de los tres vaciados (1995, 1996 y 1997). Se ha adoptado una escala horaria relativa, tomando como cero un momento previo a la llegada de las primeras aguas del embalse. Una línea discontinua (en el caso de 1995) indica la evolución orientativa seguida por los caudales salientes. El momento de llegada del frente de agua se indica con una "A" y la llegada del poso final de Iodos con una "L". La parte final del primer vaciado se caracterizó por una serie de pulsos alternos de caudal líquido y caudal sólido debido al taponamiento y destaponamiento continuado de los conductos de los desagües de fondo.

La llegada del frente de agua determinó siempre un aumento del contenido en oxígeno hasta el $100 \%$ de saturación lo que, teniendo en cuenta el origen profundo de las primeras aguas salientes, es atribuible a varios factores (condición trófica del embalse, mezcla vertical de su columna de agua, aireación de las propias aguas salientes, etc.)

El oxígeno disuelto se mantenía alrededor del $100 \%$ de saturación hasta la salida de lodos, cuando tenía lugar un descenso hasta concentraciones mínimas en muy pocos minutos. En el primer vaciado se alcanzó la situación de anoxia que se mantuvo, con pequeños incrementos transitorios (+ $3 \%$ saturación), entre 4 y 5 días después del vaciado, cuando se registró una clara tendencia a la recuperación de la normalidad (50-60\% de saturación 4 días después).

En el segundo vaciado ya no se alcanzó la anoxia absoluta (mínimo $0.05 \mathrm{mg} \mathrm{O} / 1 \mathrm{y}$, además, ésta sólo estuvo por debajo del $1 \%$ de saturación durante unos 38 minutos, con una rápida recuperación en las horas siguientes. En el tercer vaciado, el descenso del oxígeno con la salida de lodos fue algo más gradual y en ningún caso estuvo por debajo del $1 \%$ de saturación. El periodo de mínimo contenido de oxígeno fue más amplio que el del segundo vaciado, pero la recuperación posterior fue también mucho más notable $(40 \%$ a las 9 horas y más del $60 \%$ unas 10 horas más tarde.

En líneas generales, el comportamiento del oxígeno disuelto durante los tres vaciados fue muy similar, a excepción de lo ocurrido tras la salida de los lodos, que ya ha sido comentado. Al igual que con la temperatura, en todas las figuras se puede observar una tendencia hacia la regularización de los registros desde 1995 a 1997. Este hecho, además de estar relacionado con lo indicado para la temperatura, también tiene que ver con la progresiva liberación de carga orgánica que ha supuesto para el embalse la salida de lodos, la aireación de sedimentos con la atmósfera, el lavado y lixiviado de los sedimentos con la cubeta vacía. Lógicamente, también ha influido la eliminación reiterada de toda la población biológica de la columna de agua, desde el plancton hasta los peces, dando lugar a los mínimos poblacionales en el momento del tercer vaciado.

En E-5 la llegada de los frentes de agua durante los tres vaciados no supuso una variación significativa del contenido de oxígeno, que se mantuvo en valores normales hasta la llegada de los Iodos.

Lógicamente todo el cauce del río Esera aguas abajo de la presa se comportó como un decantador, de manera que hasta que no estuvo saturado de lodos el agua asociada a ellos podía circular. Ello explica que el descenso del contenido de oxígeno hasta valores de anoxia se observara 

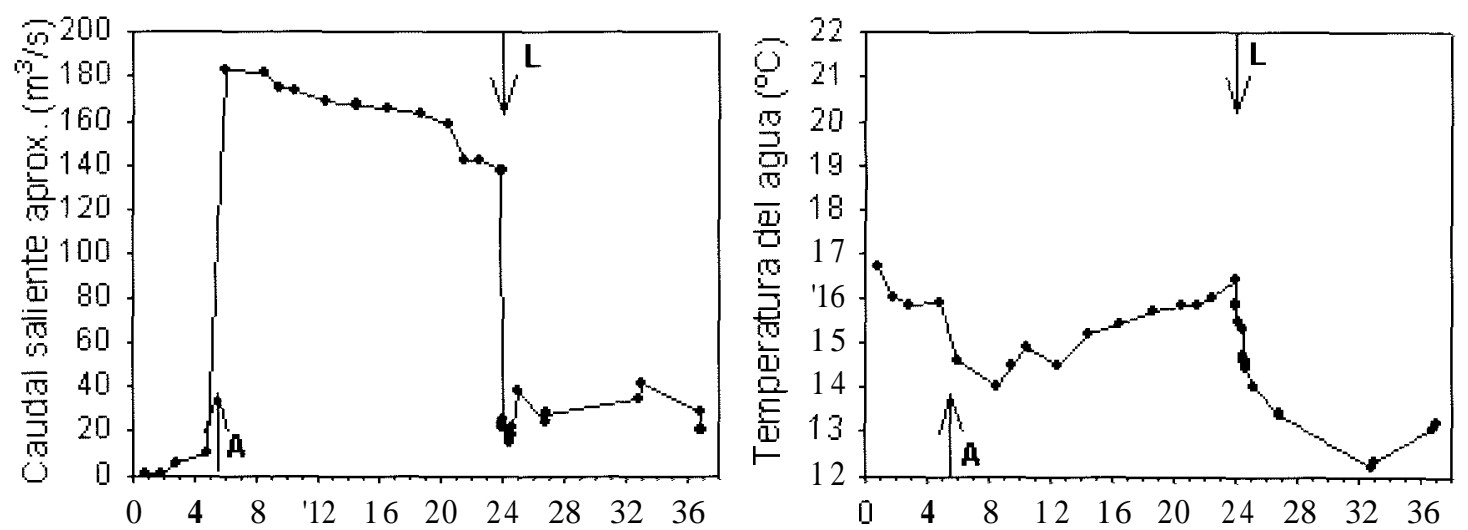

Horas respecto al inicio del vaciadu (1995)

Hnras respecto al inicio del vaciado (1995)
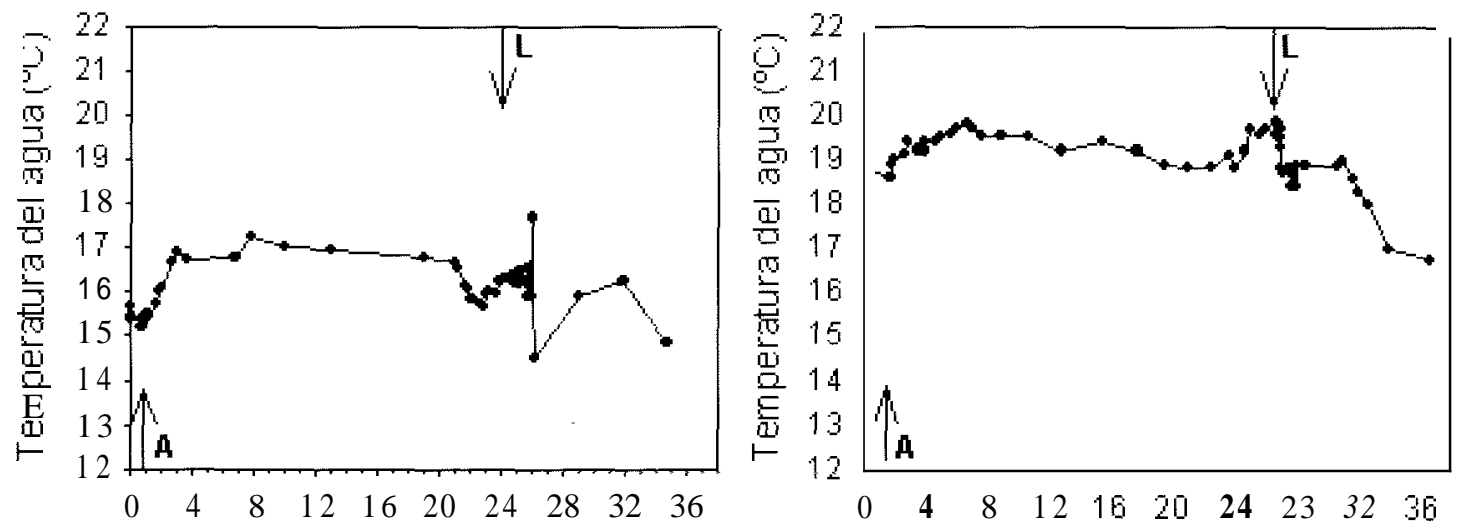

Hnras respecto al inicio del vaciado (1996)

Hnras respecto al inicio del vaciadu (1997)

Figura 2. Evolución temporal de los caudales salientes del embalse durante el vaciado de 1995 (superior izquierda). Evolución temporal de la temperatura del agua en el río Ésera unos 2.50 in por debajo de la presa (punto E-3) durante los tres vaciados de 1995 (superior derecha). 1996 y 1997 (inferior). Temporal evolution of oufflow from the reservoir during the dam flushing of 1995 (upper left). Temporal evolution of water temperature ir7 the Esera river $250 \mathrm{~m}$ downstream the dam (sampling place E-3) during the dam flushing of 1995 (upper right), 1996 and $1997($ down).

siempre con un cierto decalaje (hasta unas 4-5 horas más tarde en el primer vaciado) de la llegada del poso de lodo, probablemente en el momento en el que el cauce estaba absolutamente colmatado. En el primer vaciado se alcanzó la anoxia y se mantuvo alrededor de 1 día, siendo necesarios unos 3 días para que los contenidos de oxígeno se situaran alrededor del $20 \%$ y un día mas (4 días) para que se alcanzasen valores por encima del $70 \%$ de saturación. En el segundo y tercer vaciados, no se llegó nunca a la anoxia y la dura- ción del periodo con mínimas concentraciones de oxígeno fue muy inferior (1-2 días).

Dada la importancia del contenido de oxígeno disuelto para las comunidades acuáticas, hay que destacar que en el río Cínca, durante los días en que tuvieron lugar los vaciados y los posteriores a estos, en ninguno de los puntos muestreados a lo largo de los tres vaciados, se registraron concentraciones de oxígeno inferiores a los $4 \mathrm{mg} / \mathrm{l}$. El valor mínimo fue de $4.7 \mathrm{mg} \mathrm{O} / 1$ medido entre 2 y 3 días después del primer vaciado cerca de la 

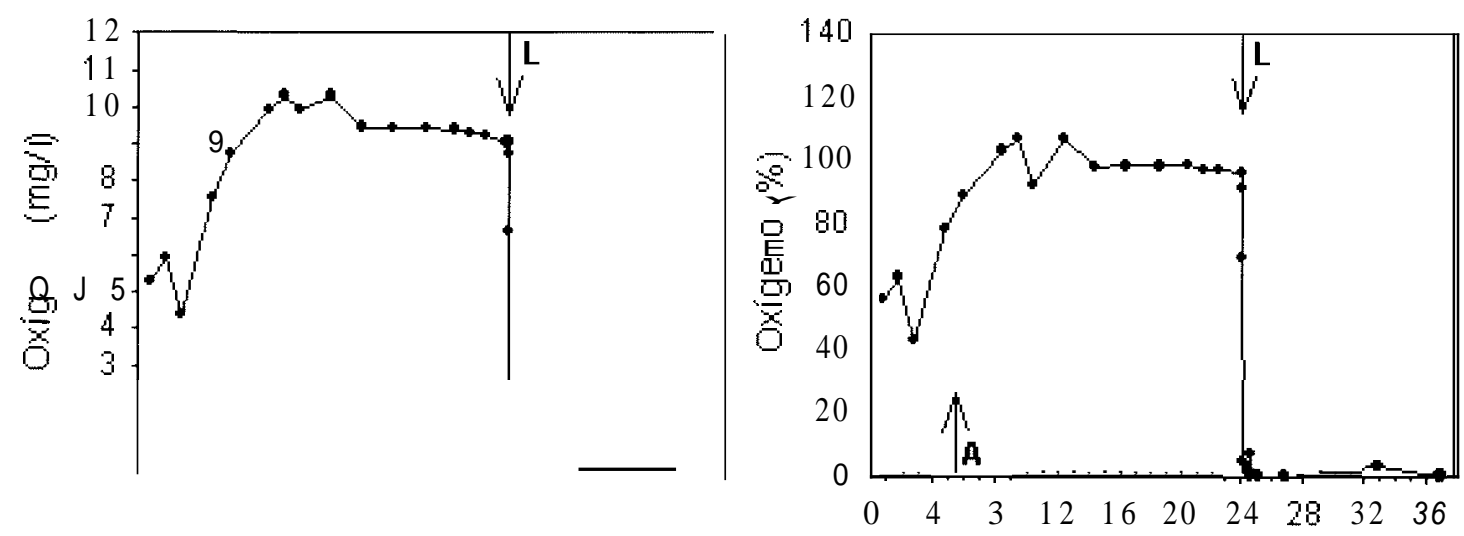

Horas respecto al inicio del vaciado (1995)
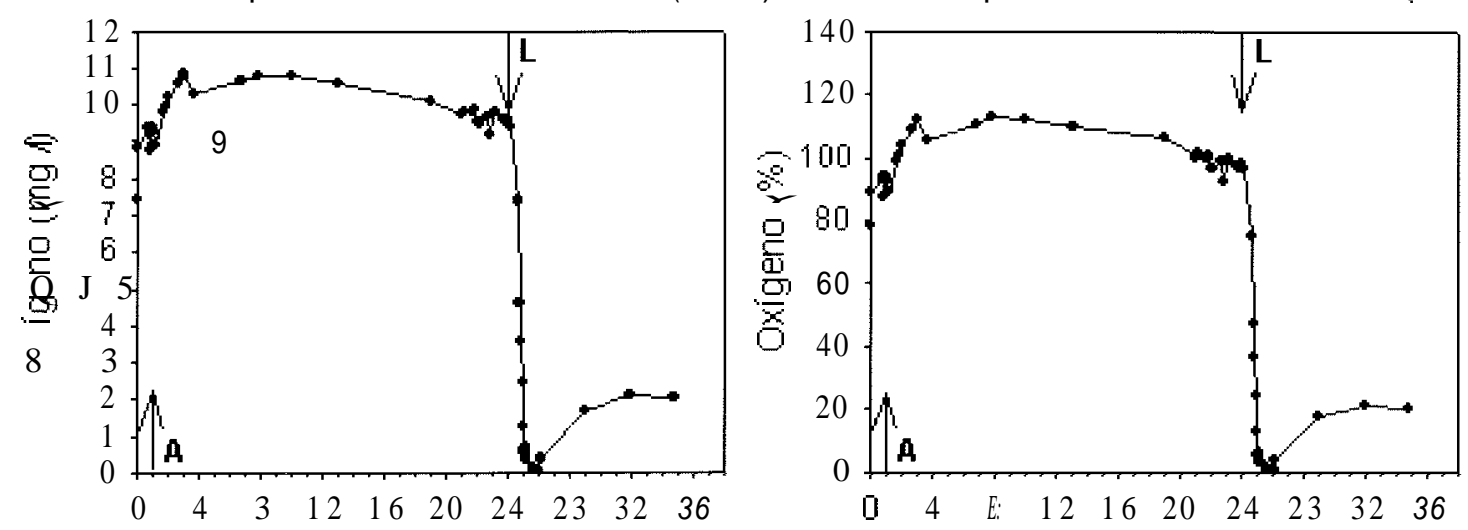

Horas respecto al inicio del vaciado (1996)
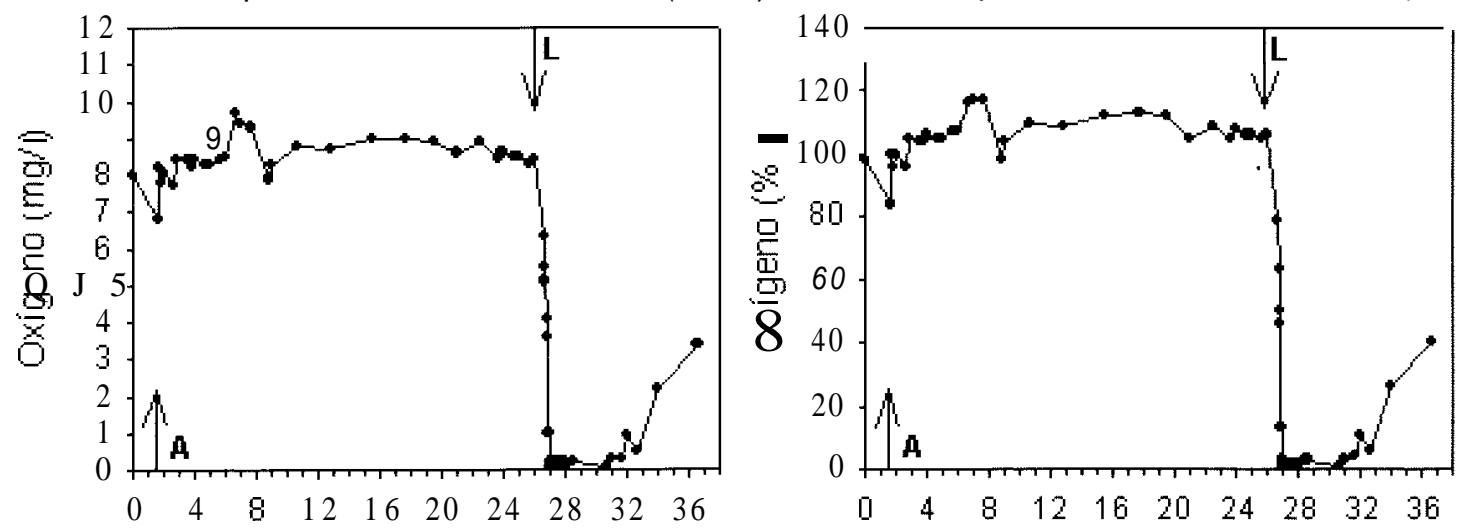

Horas respecto al inicio del vaciado (1997)
Horas respecto al inicio del vaciado (1997)

Figura 3. Evolución temporal de la concentración de oxígeno disuelto (izquierda) y del tanto por ciento de saturación de oxígeno (derecha) en el río Ésera unos $250 \mathrm{~m}$ por debajo de la presa (punto E-3) durante los tres vaciados de 1995, 1996 y 1997. Temporal evolution of dissolved oxygen concentration and saturation in the Esera river $250 \mathrm{~m}$ downstream the dam (sampling place E-3) during the dam fluching of 1995 , 1996 and 1997. 
desembocadura del río Esera, en la orilla más influida por la entrada de las aguas con lodo. En el primer vaciado, el $97 \%$ de las determinaciones de oxígeno disuelto realizadas en el río Cínca a la altura del Puente de las Pilas se situaron por encima de $5 \mathrm{mg} / \mathrm{l}$, el $91 \%$ por encima de $7 \mathrm{mg} / \mathrm{l}$ y el $55 \%$ por encima de los $9 \mathrm{mg} / \mathrm{l}$.

En cuanto a la temperatura del agua. con la salida de los Iodos se detectó siempre un descenso muy brusco y acusado (unos $2^{\circ} \mathrm{C}$ en pocos minutos en 1995 y unos $3^{\circ} \mathrm{C}$ en 1996), alcanzandose en general los valores mínimos absolutos unas horas más tarde, cuando probablemente buena parte del agua circulante aguas abajo de la presa era el agua intersticial lixiviada de los sedimentos aún retenidos en el interior del embalse y mezclada con la de los ríos entrantes (a menos temperatura) (Fig.2).

En términos comparativos, se observa que a lo largo de los tres años la temperatura del agua durante los vaciados tendió a disminuir su variabilidad y a presentar unos valores medios mayores. Tanto uno como otro aspecto son debidos al estado de estratificación del embalse antes del vaciado, al volumen de agua embalsado y a su temperatura media.

El rasgo más destacable de la evolución del pH del agua, durante los tres vaciados y tanto en E-3 como en E-5, fue su moderado intervalo de variación, entre 7.0 y 8.4 .

La evolución seguida por la conductividad eléctrica del agua durante los vaciados fue muy distinta en el primer año (1995) respecto a los otros dos (1996 y 1997) (Fig. 4). En 1995 la llegada del frente de agua supuso una clara disminución de la conductividad en relación con los valores típicos de las aguas estancadas en el río Esera; esta situación se mantuvo hasta el momento de la llegada de los lodos, cuando la conductividad aumentó de forma espectacular después de presentar una fluctuación relacionable con los cambios en la proporción agua-lodo del caudal saliente del embalse. El intervalo de valores normales (300-450 $\mu \mathrm{S} / \mathrm{cm})$ se recuperó unos 4 días más tarde.

Por el contrario, en 1996 y 1997 la conductividad del agua apenas experimentó algún cambio en el inicio del vaciado, manteniéndose hasta el final en un rango de variación muy estrecho. Con el frente de lodos, el aumento de conductividad eléctrica del agua fue manifiesto, pero sin llegar a los valores máximos registrados en 199.5, lo que debe poder interpretarse como que el primer vaciado fue el que movilizó más sedimento y el de más carga mineral y orgánica.

En E-5 ocurrió prácticamente lo mismo que en E-3 sólo que de una forma más atenuada, sin alcanzarse valores de conductividad máximos tan elevados en ningún caso. Tal y como ocurría con el oxígeno el efecto decantador del cauce del río Ésera aguas abajo de la presa hizo que el frente inicial de lodo llegara a E-5 con una carga más baja de sólidos en suspensión (mayor dilución, menor conductividad) de manera que los valores más altos de este parámetro se registraron varias horas más tarde.

La dinámica seguida por las concentraciones de amonio en E-3 (y también en E-5), fue claramente antagónica a la del oxígeno disuelto, hecho bien visible si se comparan las figuras correspondientes (Figs. 3 y 5 ).

En E-3 el amonio alcanzó en 1995 una primera punta con las primeras aguas salientes por filtración del embalse (caudales reducidos), tal y como ocurrió con el pH. La llegada del frente de agua supuso una disminución, lo que confirma el carácter trófico indicado para el embalse, no observándose un aumento significativo hasta el momento de la llegada del frente de lodos, cuando se alcanzaron concentraciones del orden de $3.5 \mathrm{mg} / \mathrm{l}$.

En 1996 y 1997, se llevó a cabo un seguimiento mucho más intensivo (mucho más frecuente) del amonio para acotar mejor su evolución, observándose lo que es habitual para esta forma de nitrógeno inorgánico disuelto en los sistemas acuáticos. Se trata de la alternancia de puntas registrada cerca del final del vaciado en 1996 y durante todo el proceso en 1997.

Durante los dos Últimos vaciados, las concentraciones máximas fueron algo (1996) o muy (1997) inferiores a las de 199.5. En $1996 \mathrm{el} \mathrm{amo-}$ nio se mantuvo en concentraciones bajas durante todo el proceso, destacando una punta registrada varias horas después del final del vaciado. La variabilidad del amonio en 1997 fue muy elevada durante todo el vaciado, intensificándose con la salida del poso final de lodos. Sobre este particular cabe indicar que otro parámetro de alta sensibilidad como es el oxígeno disuelto, también presentó una mayor variabilidad durante el vaciado de 1997. Esto puede interpretarse como que en 

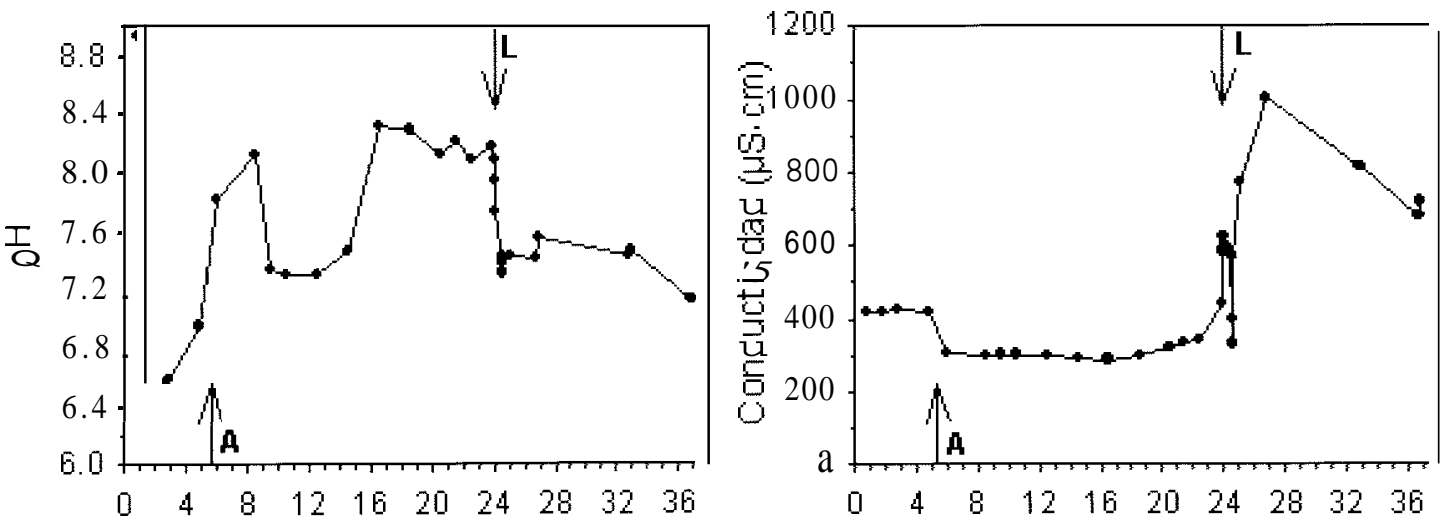

Horas respecto al inicio del vaciado (1995) Horas respecto al inicio del vaciado (1995)
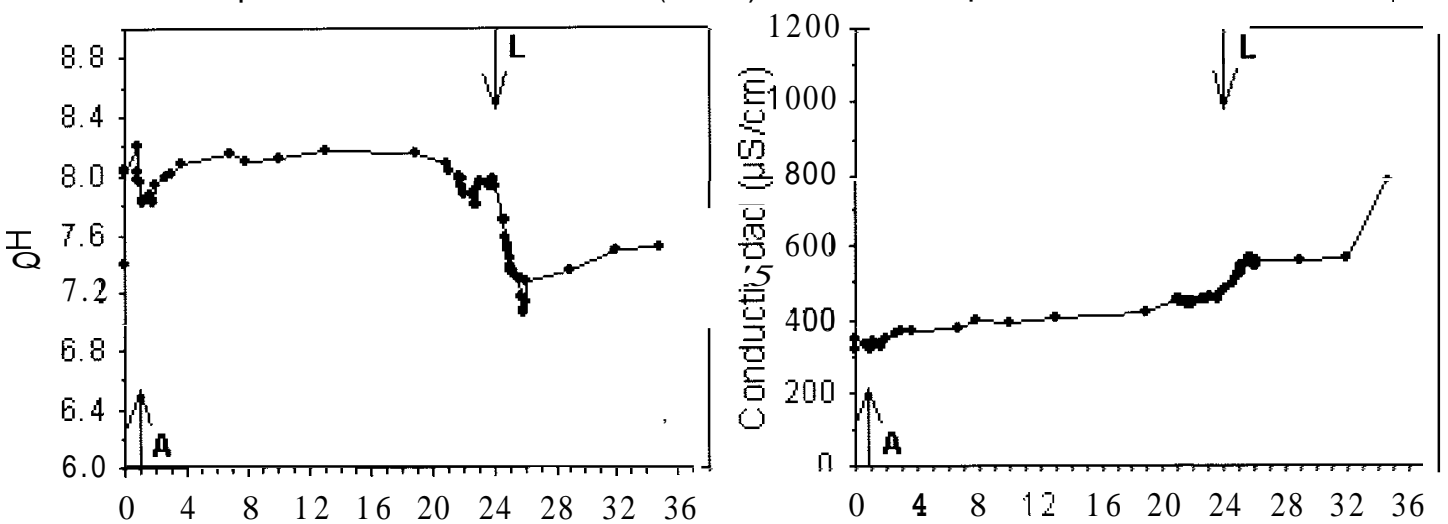

Horas respecto al inicio del vaciado (1996) Horas respecto al inicio del vaciado (1996)
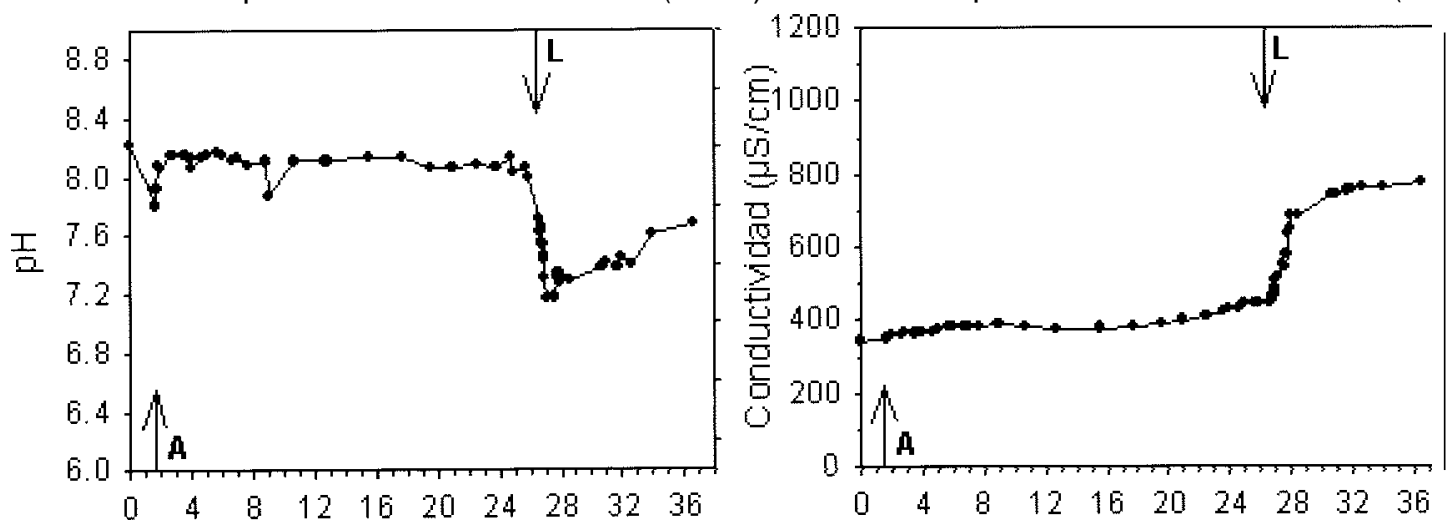

Horas respecto al inicio del vaciado (1997) Horas respecto al inicio del vaciado (1997)

Figura 4. Evolución temporal del pH del agua (izquierda) y la conductividad eléctrica (derecha) en el río Ésera unos $250 \mathrm{~m}$ por debajo de la presa (punto E-3) durante los tres vaciados de 1995,1996 y 1997. Temporal evolution of pH of water (left) and electric conductivity (right) in the Esera river $250 \mathrm{~m}$ downstream the dam (sampling place E-3) during the dam flushing of 1995, 1996 and 1997. 
este tercer y último vaciado, el sedimento salió de una forma más gradual en el tiempo y no concentrado al final. De hecho, en 1997 el poso final de lodos fue claramente más diluido que el de los dos años anteriores, lo que sin duda redundó en la mayor proporción de ejemplares de peces vivos salientes del embalse.

En ningún momento, por el $\mathrm{pH}$ y la temperatura del agua, se alcanzaron concentraciones de amoníaco mayores de $0.03 \mathrm{mg} / \mathrm{L} \mathrm{NH}$,

En E-5 el amonio se comportó con un decalaje respecto a lo ocurrido en E-3, presentando unos valores máximos más bajos que en E-3 y con unas horas de retraso respecto a la llegada del frente de lodos, lo que se explicaría por lo comentado en relación a la conductiviáad eléctrica y el oxígeno disuelto, es decir, por el efecto decantador del cauce del Ésera y el desfase que ello supuso en la aparición de las máximas concentraciones de lodo en E-5. En el río Cínca las concentraciones máximas de nitrógeno en forma de amonio se situaron alrededor de los 1.4 $\mathrm{mg} / \mathrm{l}$.

Entre los metales, lo más destacable fue la concentración de plomo asociada a la salida del agua durante el primer vaciado $(0.12 \mathrm{mg} / \mathrm{l})$, y aún más a la salida de los lodos durante el segundo y tercer vaciados cuando las concentraciones punta fueron incluso algo superiores $(0.18 \mathrm{mg} / \mathrm{l}$ y 0.14 $\mathrm{mg} / \mathrm{l}$, respectivamente).

El cadmio alcanzó durante los tres vaciados, unas concentraciones máximas entre 0.04 y 0.06 $\mathrm{mg} / \mathrm{l}$. Sobre el plomo y el cadmio cabe destacar que se superaron entre 4 y 8 veces respectivamente los valores máximos tolerables para aguas potables en España, si bien en ambos casos los efectos de toxicidad en peces se manifiestan por encima de $1 \mathrm{mg} / \mathrm{l}$ (Rodier, 1981). Tal y como se ha comentado para otros parámetros, en el río Cínca los cambios físicos y químicos derivados de los vaciados se manifestaban significativamente atenuados. Así, en el caso del plomo y el cadmio se alcanzaron concentraciones máximas en ambos casos de unos $0.06 \mathrm{mg} / \mathrm{l}$, claramente inferiores a las de E-3 para el plomo y más o menos del mismo orden para el cadmio. Hay que matizar que en el río Cínca a la altura de la desembocadura del río Vero (punto C-4) las concentraciones de estos dos metales pesados a lo largo de todo el seguimiento de los vaciados, permanecieron en valores no detectables.

\section{EVOLUCIÓN CUALITATIVA DE LA COMUNIDAD DE PECES DURANTE LOS VACIADOS}

Sin duda, el aspecto mas espectacular de los tres vaciados del embalse de Barasona, al margen de la cantidad de lodos salida en 1995, ha sido el arrastre de peces producido al final de los vaciados. Por otro lado, la comunidad de peces era en buena medida uno de los principales motivos de conservación (por diferentes causas) y por tanto un referente obligado en el estudio de seguimiento realizado.

La figura 5 pretende ilustrar. sin ninguna pretensión cuantitativa y de forma comparada, las tendencias observadas "in situ" sobre los arrastres de peces del embalse al final de los tres vaciados. Los aspectos considerados en la comparación son la variedad de especies ícticas, la abundancia total de peces, la proporción entre especies alóctonas (introducidas) y autóctonas (del país), la variedad de tamaños, el tamaño medio y la proporción de ejemplares muertos.

En los términos cualitativos y comparativos expuestos, y a modo de síntesis global para todos los aspectos considerados, puede indicarse que a lo largo de los tres vaciados sucesivos se produjo una disminución de la variedad de especies que alcanzó su mínimo en 1996 cuando prácticamente toda la población íctica saliente del embalse eran carpas. En 1997 se produjo una recuperación de la variedad de especies debido a una mayor presencia de las autóctonas (barbo, madrilla). El número total de individuos arrastrados fue máximo en 1996 y mínimo al año siguiente, lo que demuestra la capacidad de reproducción de la carpa (especie dominante en 1996) y, una vez desaparecida esta (o reducida a mínimos), el mayor tiempo requerido por las especies autóctonas para recolonizar el embalse.

Teniendo en cuenta la dominancia absoluta de la carpa en 1996, la proporción entre especies alóctonas y autóctonas fue máxima en ese año. En 1997 el embalse quedó constituido casi exclusivamente por especies del país, aspecto este importante por varios motivos. El primero por que supone un empezar de nuevo en la gestión piscícola del embalse y por tanto una nueva oportunidad de no llevarlo al estado de colonización por especies exóticas que presentaba antes de los vaciados. En segundo lugar, hay que des- 
tacar de forma especial la erradicación conseguida con la carpa en 1996, que se tradujo en 1997 en cambios muy interesantes en el embalse durante 1997, como el aumento de la transparencia del agua (la carpa es un gran removedor de lodos y por tanto un factor de enturbiamiento del agua muy significativos en el caso de grandes densidades). Este aumento de la transparencia del agua redundó en la reaparición de carófitos en las zonas con luz del fondo del embalse (zona litoral) y también en un aumento muy significativo de la población de anfibios, por citar los cambios más evidentes.

En cuanto a la variedad de tamaños, lo más interesante fue la uniformidad detectada en los peces del embalse en 1996 (carpas) que hizo sospechar incluso la posibilidad de que Barasona se hubiera repoblado de forma ilegal con esa especie entre el vaciado de 1995 y el de 1996. Esta interpretación es aun mantenida por algunos especialistas y técnicos relacionados con el seguimiento ambiental de los tres vaciados, pero parece ser que no es imposible que la explosión demográfica de carpas detectada en 1996 pudiera haberse debido a una recuperación natural de esa especie a partir de las puestas de la primavera de 1995 que quedaron en el lodo del embalse tras el primer vaciado, junto con las de la primavera de 1996, a partir de ejemplares de carpa que se refugiaron en los tributarios del embalse. No hay que olvidar que estas puestas son, hasta cierto punto, resistentes a la desecación.

El tamaño medio de los peces disminuyó de forma espectacular entre 1995 por un lado y 1996 y 1997 por otro, lo cual es lógico si se tiene en cuenta que en los dos últimos años, las poblaciones de peces procedían de puestas recientes. Hay que indicar que en el primer vaciado salieron del embalse ejemplares de una talla considerable (hasta $50 \mathrm{~cm}$ aproximadamente).

Finalmente, la mortandad de los peces disminuyó de forma progresiva a lo largo de los tres vaciados, pudiendo cifrarse a titulo orientativo en un $90-95 \%$ de la población del embalse en 1995 , un $60-65 \%$ en 1996 y en un $30-35 \%$ en 1997.

\section{EVOLUCIÓN DE LOS TRAMOS FLUVIALES TRAS EL VACIADO}

\section{Sobre las características físicas y químicas del agua}

Tras los vaciados, se procedió al seguimiento de los cursos de agua para reconocer el alcance (temporal-espacial) y el sentido de los posibles cambios. Cabe indicar que en los tres años, se registraron importantes crecidas naturales en otoño e invierno, tanto en el Esera como en el Cínca, lo que si bien fue uno de los factores clave en la rápida recuperación de los ríos, también es cierto que condicionaron, hasta cierto punto, los muestreos limnológicos (en particular los de macrobentos). A continuación se comentan brevemente los resultados obtenidos más relevantes en las fases de postvaciado.

Sobre la temperatura del agua y la conductividad eléctrica, cabe indicar que siguieron las tendencias de cambio propias del ciclo anual (estacionalidad), si bien la conductividad presentó un sensible aumento generalizado en los ríos aguas abajo del embalse.

El oxígeno disuelto se consideró un parámetro clave en las fases de seguimiento postvaciado. En el río Esera y durante el primer vaciado, se registraron valores muy bajos hasta unos 3 días después, mientras que en el Cínca en ningún caso se alcanzaron valores por debajo del mínimo tolerable $(3 \mathrm{mg} / \mathrm{l})$ y sólo en dos ocasiones por debajo del mínimo recomendable $(5 \mathrm{mg} / \mathrm{l})$. En general, en pocos días después de los vaciados (2-3) se alcanzaban concentraciones más o menos normales, alrededor de los 8-10 mg/l, con una cierta variabilidad entre muestras, posiblemente atribuible a cambios más o menos aleatorios en la carga de sólidos en suspensión en el agua, especialmente en el río Esera.

Estos mismos cambios en la carga de lodos explican porque la distribución de las concentraciones de amonio en los periodos siguientes a los vaciados no siguiera un patrón de variación claro. Parece claro que en su circulación por el interior del embalse, las aguas entrantes aumentaban su contenido en amonio, si, bien hasta niveles poco reseñables tanto en el Esera como en el Cínca aguas abajo.

La baja carga orgánica de los lodos de Barasona se pone de manifiesto, en la ausencia de valores reseñables de DQO a lo largo del pro- 

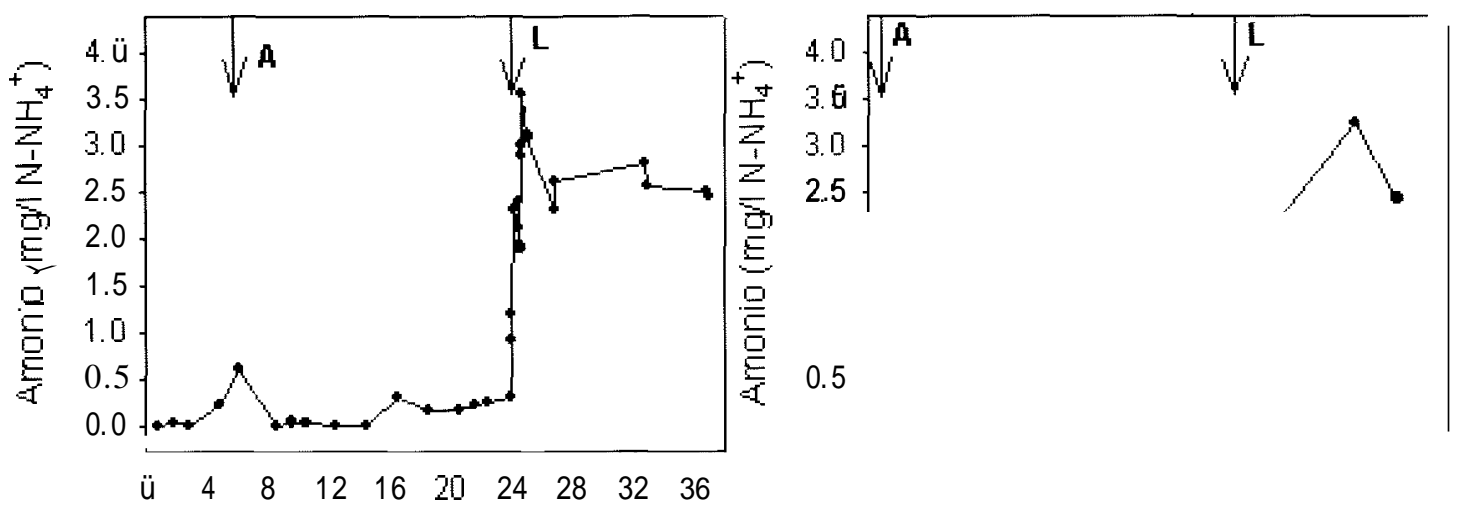

Horas respecto al inicio del vaciado (1995)

Horas respecto al inicio del vaciado (1996)
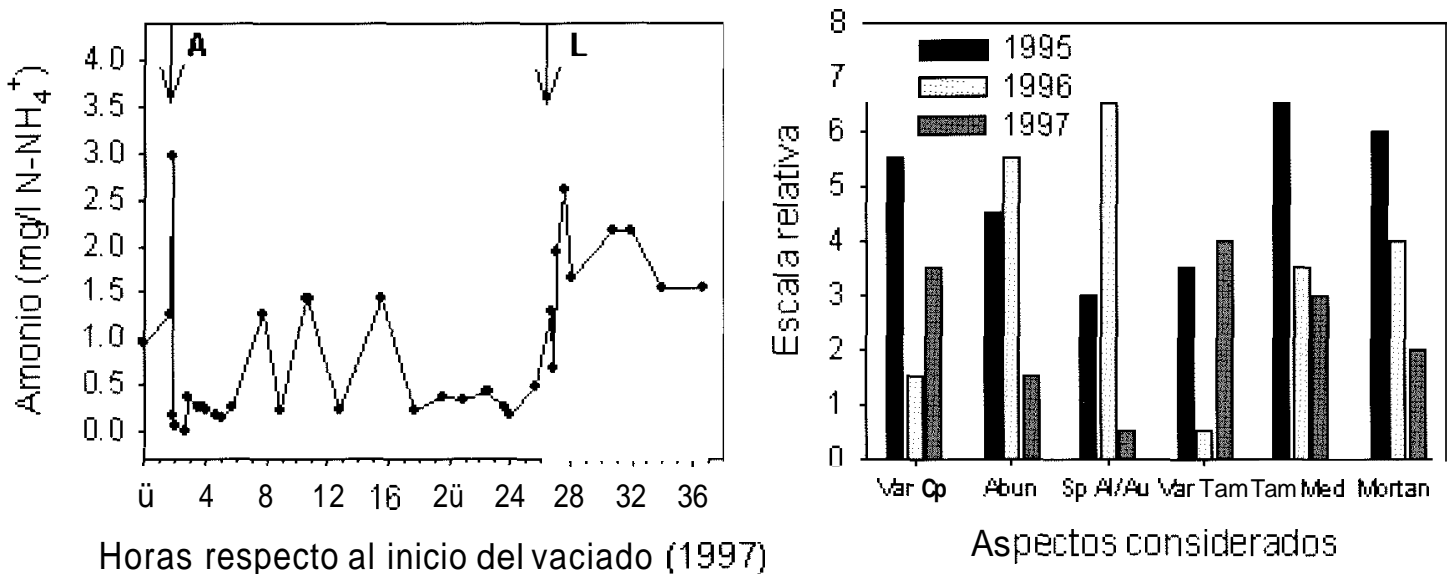

Horas respecto al inicio del vaciado (1997)

Figura 5. Evolución temporal de la concentración de nitrógeno en forma de amoiiio durante los vaciados de 1995 y 1996 (superior) y durante el de 1997 (inferior) en el río Ésera unos 250 m por debajo de la presa (punto E-3). Representación orientativa de la evolución de una serie de descriptores de la población piscícola saliente del embalse, durante los tres vaciados de 1995, 1996 y 1997 (inferior). (Var Sp: variedad de especies; Abund: abundancia: Sp Al/Au: proporción de especies alóctonas y autóctonas; Var Tam: variedad de tamaños; Tam Med: tamaño medio; Mortan: grado de mortandad observada). Temporal evolution of ammonia concentration in tlie Esera river $250 \mathrm{~m}$ downstream the dam (sampling place E3) during the dam fluching of tlie reservoir in 1995, 1996 (up) and 1997 (down). Guiding figure showing temporal evolution of some aspects of fish population outstanding from reservoir during its total emptying in 1995, 1996 arid 1997 (down). (Var Sp: species variety; Abund: abundance; Sp Al/Au: relationship between exotic and native species; Var Tam: size variety; Tam Med: mean size; Mortan: apparent death rate).

ceso de postvaciado. Un aspecto sobre el que las circunstancias del vaciado hicieron pensar, fue cuál podría ser el efecto sobre la carga orgánica de los ríos, la descomposición de la cantidad de peces muertos que no se pudieron retirar de los cauces. Las crecidas registradas durante el postvaciado fueron dispersando este aporte adicional de materia orgánica y diluyéndolo, hasta el punto que no se observaron ni valores altos de DQO ni de amonio, como producto de descomposición.

El hierro y el manganeso son, como el amonio, dos elementos indicadores en este caso de la existencia de unas condiciones de oxido-reducción favorables a la solubilización de compuestos y elementos desde el sedimento. Ambos aparecen en concentraciones nulas o muy bajas en aguas naturales bien oxigenadas. Los valores encontrados 
en la mayoría de casos durante el postvaciado son nulos (concentraciones no detectables) o muy bajos, tal y como ocurría antes del vaciado.

\section{La comunidad de macroinvertebrados bentó- nicos}

A partir de los inventarios de la comunidad macrobentónica realizados tras el vaciado, se han podido extraer conclusiones bastante precisas sobre la recuperación de los tramos de río situados aguas abajo del embalse.

Los puntos seleccionados para evaluar la tendencia y la capacidad de recuperación han sido E5 y C-4 (Fig. 1) junto con algunas muestras puntuales realizadas en el Cínca a la altura de Monzón, en Albalate, en Zaidín y en el río Segre después de la desembocadura del Cínca (Granja d'Escarp).

El punto de muestreo E- 1 se ha utilizado como referencia con el fin de poder discriminar posibles variaciones no atribuibles a los efectos del vaciado.

Cabe destacar que unos 4 ó 5 días después del vaciado se procedió a la realización de una inspección visual de los tramos de río comprendidos entre la Presa de Barasona y Monzón, constatándose la presencia de macroinvertebrados en las zonas marginales del cauce (las únicas en las que se podía muestrear como consecuencia del elevado caudal y turbidez del agua) en varios puntos.

En general, las campañas de Mayo a Julio de 1996 y de 1997, unos 6 meses después del cierre del embalse tras los vaciados de 1995 y 1996, han permitido observar la clara y evidente recuperación de los tramos de río estudiados, obteniéndose unos datos muy similares a los de las campañas de Mayo a Julio de 1995, previas al vaciado. La Tabla 2 muestra una síntesis de los resultados obtenidos.

Respecto a los efectos del vaciado sobre el número de unidades sistemáticas determinadas en cada punto cabe destacar que la recuperación de los valores habituales anteriores al vaciado fue manifiesta.

En los resultados encontrados en cuanto a biomasa, densidad y diversidad, no se observó la existencia de valores del postvaciado que quedasen fuera (por debajo) del intervalo de variación natural, con excepción de la diversidad en el punto E-5.
Comparando los valores medios de densidad y biomasa antes y después del vaciado, se deduce que el macrobentos en las campañas posteriores se ha caracterizado por altas densidades y bajas biomasas. Esta situación es lógica si se tiene en cuenta que las especies dominantes después del vaciado son polivoltinas y de pequeño tamaño.

En 1996, tras el primer vaciado, la composición del macrobentos en el río Esera (E-5) varió al disminuir la representación de las unidades sistemáticas poco tolerantes, tanto en términos de variedad como de abundancias, mientras que aumentó el de las más tolerantes. Esta situación. aunque puede atribuirse a los efectos del vaciado, también se detectó en E-1, punto de muestreo no afectado por el vaciado. La situación fue distinta en 1997 cuando en este caso en el punto E-3 (el más afectado) se encontró una variedad y abundancia de unidades sistemáticas de macrobentos reseñables (Polycentropidae, Hydropsyche, Ecdyonurus, Baetis, Chironomidae, Simuliidae, ELmidae y Lymnaeidae) con algunas unidades sistemáticas hasta la fecha nunca identificadas en ese lugar y con un claro valor indicador de mejora de las condiciones del cauce.

Tanto en el río Esera (E-5) como en el Cínca (C-4) el hábitat varió poco en relación al macrobentos. Después de los vaciados se observó el mantenimiento de la dominancia del macrobentos reófilo en todos los puntos, aunque con abundancias menores y con un sensible incremento de las presencia y/o abundancia de las unidades sistemáticas más tolerantes, tal y como ya se ha indicado. También aumentó la representación de especies asociadas a la presencia de fitobentos (productores primarios acuáticos), lo que en términos de teoría ecológica simple, es coherente con el aumento de productividad que, como respuesta de cualquier sistema, sigue a las perturbaciones de carácter reversible que no han desbordado las capacidades del sistema.

En efecto, una vez eliminados los depósitos de lodos del cauce, la constancia del caudal junto con la sensible eliminación de vegetación de ribera, suponen una serie de cambios que se traducen en un aumento de la productividad del medio acuático.

En cuanto a estrategias de nutrición lo más destacable es la disminución de los detritívoros, (coherente con el sentido de los cambios detectados a nivel de hábitat), y el aumento de la representación de trituradores en términos de abundan- 
cia, que también sería coherente con una mayor producción primaria en el cauce. Estos cambios en cuanto a la distribución de las estrategias de nutrición, son prácticamente explicables en su totalidad por los cambios de densidades entre las tres unidades sistemáticas dominantes (Baetidae y Chironomidae, ambas trituradoras, y por otro lado Hydropsychidae, filtradora). Al tratarse de especies polivoltinas sus variaciones de densidad pueden ser notables y rápidas, modificando de forma significativa los porcentajes de representación del conjunto de estrategias de nutrición.

Sobre la capacidad de resistencia de estas tres unidades sistemáticas, basta con decir que en una inspección cualitativa realizada unos días después del primer vaciado ya se detectó su presencia en la zona del Puente de las Pilas y en el punto $\mathrm{C}-4$, junto con algunos Oligoquetos y larvas de cangrejo rojo, por lo que cabe suponer que persistieron durante el vaciado.

Dos meses más tarde (Diciembre de 1995) se observaron prácticamente los mismos grupos, mientras que en Junio de 1996 se detectó el mismo número de unidades sistemáticas que en Junio de 1995.

En el río Cínca (C-4) la similitud entre las estrategias de nutrición antes y después del vaciado siempre fue muy alta.

En general los puntos de muestreo, cuanto más alejados de Barasona, menor ha sido el grado de alteración sufrido y/o más rápida su recuperación. Tal es el caso del río Cínca en Monzón, Albalate y Zaidín, así como el Segre en la Granja d'Escarp.

En cuanto a la calidad biótica, los valores del BMWP' posteriores a los vaciados caen todos ellos dentro del intervalo de variación normal de los valores encontrados antes. Curiosamente el punto que más variabilidad y cambios mostró en este sentido fue el de referencia (E-1) situado aguas arriba del embalse.

\section{CONCLUSIONES}

Los cambios más importantes en cuanto a calidad del agua han ocurrido durante el primer vaciado y concretamente en el momento de la llegada de los lodos, en la zona próxima aguas abajo de la presa. Se observó una notable atenuación en el tramo final del Esera y en algunos casos la ausencia de efectos reseñables en la química del agua del río Cinca a partir del Puente de las Pilas. La magnitud de los cambios se ha ido atenuando también en los sucesivos vaciados, al igual que la duración de la fase más crítica, desde 4-5 días en el primero vaciado a 1-2 días en el Último.

La magnitud y alcance de los cambios pueden ser para buena parte de los parámetros físicos, químicos y biológicos estudiados, comparables a los de una gran crecida, si bien con patrones de variación distintos. Los diferentes registros de mediciones y concentraciones no fueron en ningún caso preocupantes, hecho ligado a un aspecto fundamental del estudio y muy característico del embalse de Barasona: la baja carga orgánica presente en los sedimentos del embalse y su estado de oxidación.

De acuerdo con los resultados pueden establecerse varios bloques de conclusiones. En primer lugar, se constata la existencia de una serie de

Tabla II. Valores comparados de densidad, biomasa y diversidad antes y después de los vaciados (U.S.: Unidades sistematicas). Comparison between the values of density, biomass and diversity before and after the emptying processes (U.S.: Sistematic units).

\begin{tabular}{|c|c|c|c|c|c|c|}
\hline Estación & fecha & $n^{\circ}$ u.s. & $\begin{array}{c}\text { densidad } \\
\text { (Ind } / \mathrm{m} 2)\end{array}$ & $\begin{array}{l}\text { biomasa } \\
(\mathrm{g} \mathrm{C} / \mathrm{m} 2)\end{array}$ & $\begin{array}{l}\text { diversidad } \\
\text { (bits/ind.) }\end{array}$ & bmwp' \\
\hline E-1 & Antes vaciados & $10-13$ & $222-9844$ & $0.10-2.76$ & $0.28-0.41$ & $19-94$ \\
\hline E-1 & Ma-Jl 1995 & $10-11$ & $789-9844$ & $0.46-1.44$ & $0.28-0.34$ & $68-81$ \\
\hline E-1 & Ma-Jl 1996-97 & 9 & 578 & 0,11 & 0.34 & 48 \\
\hline E-5 & Antes vaciados & $5-8$ & $211-2155$ & $0.03-1.05$ & $0.28-0.39$ & $25-95$ \\
\hline E-5 & Ma-Jl 1995 & $5-7$ & $211-678$ & $0.03-0.30$ & $0.28-0.32$ & $25-44$ \\
\hline E-5 & Ma-Jl 1996-97 & $7-9$ & $1565-2555$ & $0.14-0.98$ & $0.26-0.35$ & $43-90$ \\
\hline$c-4$ & Antes vaciados & $10-11$ & $244-5647$ & $0.04-5.70$ & $0.29-0.40$ & $34-80$ \\
\hline$c-4$ & Ma-Jl 1995 & $10-11$ & $244-789$ & $0.04-0.40$ & $0.29-0.36$ & $34-66$ \\
\hline$c-4$ & Ma-Jl 1996-97 & $11-13$ & $2210-2711$ & $0.72-2.52$ & $0.29-0.33$ & $55-88$ \\
\hline
\end{tabular}


aspectos clave en la manifestación y consecuencias de los cambios derivables del vaciado de un gran embalse. Algunos están asociados a las características de los sistemas donde tiene lugar la acción, es decir del embalse (estado trófico, características físicas y químicas de los sedimentos, grado de colmatación, gradiente vertical de la columna de agua) y de los ríos aguas abajo (caudales circulantes, aportaciones laterales, estructura del lecho). Otros aspectos en cambio están relacionados con la propia acción del vaciado (volumen embalsado inicial, época del año, capacidad de regulación de los caudales salientes).

En segundo lugar, hay una serie de parametros físicos y químicos del agua (temperatura, $\mathrm{pH}$, conductividad, oxígeno disuelto, alcalinidad total, amonio, nitrato, nitrito, fosfato, DQO) que mostraron patrones de cambio similares y claramente asociados a la dinámica del vaciado, en concreto a la salida inicial de aguas y a la del poso final de lodos. Otra serie de variables (hierro, manganeso, cadmio, cromo VI, plomo) presentaron un patrón de variación más caótico bien sea por su mayor dependencia de situaciones puntuales, o bien por la escala de trabajo aplicada en su estudio (frecuencia de muestreo y separación entre puntos de muestreo).

Todas las afecciones relacionadas con las características limnológicas estudiadas y asociadas al proceso de los vaciados, se calificaron desde un principio como absolutamente recuperables, tal y como ha ocurrido. Si bien cabe indicar que la secuencia de tres vaciados consecutivos ha condicionado (retrasado), sin duda, la progresión de los sistemas perturbados en su conjunto. Las características de las aguas y el macrobentos, son componentes que se han recuperado prácticamente en su totalidad dentro de los plazos del estudio.

Como aspectos más críticos del estudio limnológico realizado, cabe citar la falta de explicaciones definitivas hasta la fecha, de lo acontecido en los tributarios del embalse, en particular sobre el macrobentos. Se desconoce si los cambios observados en el río Esera aguas arriba de Graus han sido debidos a fenómenos naturales (crecidas registradas), artificiales (hidropuntas) o bien a un aumento de la presión de predación por parte de la comunidad piscícola que abandono el embalse durante los vaciados. En cualquier caso quizás se haya puesto de manifiesto un aspecto poco o nada estudiado sobre los embalses referido a sus posibles efectos río arriba, bien por construcción o por gestión.

Otro aspecto de interés ha sido la estrategia de dilución del frente de lodos que se ha llevado a cabo en el río Cinca, con aguas del Embalse de El Grado, especialmente durante el primer vaciado. Esta actuación, prevista en principio como una medida correctora, habría que ver hasta que punto no contribuyó a proyectar los efectos del vaciado aguas abajo y si realmente compensó el nivel de dilución alcanzado.

Con referencia a las posibilidades de gestión futura para los tramos de río estudiados a lo largo del seguimiento, sin duda lo más destacable ha sido la disponibilidad de un caudal mínimo apreciable en el Esera, a partir de la reparación de los desagües de fondo de Barasona. Esto ha permitido una sustancial mejora de la calidad del agua y del hábitat que se ha traducido en una comunidad bentónica (fito y zoobentos) mucho más completa que la existente antes del vaciado. Este hecho es la mejor evidencia del interés que tiene el empezar a abordar de forma decidida la gestión racional de los ríos regulados.

Si en el Ésera el principal motivo de alteración era la falta de caudales circulantes, en el Cinca, además de este mismo problema aguas abajo de la Presa de El Grado, habría que plantearse un proyecto de restauración global que permita recuperar para el ecosistema fluvial un componente tan importante como es la ribera. A nivel de actuaciones más puntuales, el control y depuración de vertidos residuales, requiere también una solución rápida, en especial en el caso del río Vero cuyas aguas son el paradigma del estado de contaminación que nunca se debería permitir alcanzar a ningún río.

Al margen de los aspectos más o menos concretos comentados como conclusiones, los principales hitos del estudio se centran por un lado en el interés que ha tenido el estudio de seguimiento realizado para la validación o no de la metodología de seguimiento, prevención y corrección de efectos ambientales aplicada. Por otro lado, este tipo de experiencias siempre sirven para desmitificar y aprender limnología aplicada; en este sentido puede destacarse la constatación de que el vaciado de un embalse, según sus circunstancias, tendrá más o menos afecciones, pero siempre será una perturbación de efectos reversibles, tanto 
más rápida y adecuada cuanto más y mejores medidas (naturales o artificiales) de prevención y corrección existan o puedan establecerse.

Sin dejar de exigir las garantías ambientales suficientes, hay que aprender a enmarcar los posibles efectos de las actuaciones de gestión de los sistemas naturales en base al conocimiento de la capacidad de respuesta del ecosistema que las recibe. Esta es otra conclusión, sencilla pero importante. No se trata por tanto de prevenir desde el desconocimiento, sino de perder el miedo a actuar con conocimiento.

\section{BIBLIOGRAFÍA}

ARMENGOL, J., J.L. RIERA \& J.A. MORGUI. 1991. Major ionic composition in the Spanish reservoirs. Verh. Internat. Verein. Limnol. 24: 1363-1366.

CARDINAL, H. 1989. Bilan des Recherches et Recomendations en Matière de Vidanges de Retenues: Procedure LIVRE. Direction des Études et Recherches. EDF. HE-31/88-20.

FISHER, S.G., L.J. GRAY, N.B. GRIMM \& D.E. BUSCH. 1982. Temporal Sucession in a desert stream ecosystem following flash flooding. Ecol. Monog. 52: 93-110.

GARRIC, J., B. MIGEON \& E. VINDIMIAN. 1990. Lethal effects of draining on brown trout. A predictive model based on field and laboratory studies. Wat. Res., 24: 59-65.

GRIZZLE, J.M. 1981. Effects of Hypolimnetic Discharge on Fish Health Below a Reservoir. Trans. Amer. Fish. Soc., 110: 29-43.
MARGALEF, R., D. PLANAS, J. ARMENGOL, A. VIDAL, N. PRAT, A. GUISET, J. TOJA y M. ESTRADA. 1976. Limnología de los embalses españoles. Serv. Publ. Direc. Gral. Obras Hidráulicas. MOPU. Madrid. 422 pp.

MORGUÍ, J.A., J. ARMENGOL y J.L. RIERA. 1990. Evaluación limnológica del estado de los embalses españoles: Composición iónica y nutrientes". In: Actas de las III Jornadas Españolas de Grandes Presas. Barcelona: 652-668.

PALAU, A. \& C. RIVERO. 1994. Effects of hypolimnetic discharge on some physico-chemical and biological characteristics of a river below a reservoir. Verh. Internat. Verein. Limnol., 25: 1751- 1755

PALAU, A. 1996. Evaluación de los efectos ambientales del vaciado total del embalse de Barasona (río Esera, Huesca). Estudio limnológico del sistema fluvial afectado. Informe Técnico de la Confederación Hidrográfica del Ebro. Zaragoza. 178 pp.

PEVIANI, M., Y. SACCARDO, A. CROSATO \& G.GENTILI. 1996. Natural/artificial floods connected with river habitat. Proceedings of 2 nd International Symposium on Habitat Hydraulics, vol B: $175-186$.

RODIER, J. 1981. Análisis de las Aguas. Ed. Omega, S.A. Barcelona.

STEDNICKJ, J.D. 1991. Wildland water quality sampling and analysis. Academic Press, Inc. London.

TACHET, H., M. BOURNAUD \& P. RICHOUX. 1980. Introduction à I'étude des macroinvertebrés des eaux douces. Publ. Association Française de Limnologie. Paris. 